\title{
Estudio descriptivo de infecciones osteo-articulares en niños en tiempos de Staphylococcus aureus resistente a meticilina de la comunidad (SARM-Co)
}

\author{
María T. Rosanova, Griselda Berberian, Rosa Bologna, Sandra Giménez, Claudia Sarkis, \\ Ana Buchovsky, Guadalupe Pérez-MD, Jose Pinheiro y Roberto Lede
}

\section{Descriptive study of bone and joint infections in children in times of community-acquired methicillin- resistant Staphylococcus aureus (CA-MRSA)}

Background: Bone and joint infections (BJI) are relatively common in children, and community -acquired methicillin resistant Staphylococcus aureus (CA-MRSA) is the leading cause in some countries. Aim: To evaluate epidemiological data, clinical and microbiological features and outcome of BJI. Methods: A prospective descriptive study was conducted. Results: 40 patients (p) completed the study. Bacterial cultures were positives

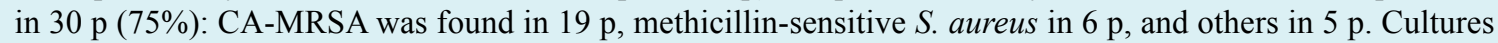
were negatives in $10 \mathrm{p} \mathrm{(25 \% ).} \mathrm{Median} \mathrm{treatment} \mathrm{duration} \mathrm{was} 28$ days (r: 21-40 d); Analyzing patients with CA-MRSA positive cultures separately, initial CRP was higher (Md $76 \mathrm{vs} 50 \mathrm{mg} / \mathrm{L}, \mathrm{p}<0.02$ ), normalization occurred later (Md 14 days vs 7days, $\mathrm{p}<0.03$ ), and duration of treatment (Md 32 days vs 23, p < 0.004) as well as hospital stay (Md 9 days vs $7, \mathrm{p}=0.12$ ) were longer. Sequelae were present in $3 \mathrm{p}$ and 1 relapsed: All of them with CA-SAMR. Conclusion: CA-MRSA was the leading cause of BJI and was associated with higher $\mathrm{CRP}$ on admission, later normalization and longer treatment duration. Complications as drainage requirement, and sequelae were common in those $\mathrm{p}$.

Key words: Community-acquired methicillin resistant Staphylococcus aureus, bone and joint infections, children.

Palabras clave: Staphylococcus aureus resistente a meticilina de la comunidad, infecciones osteoarticulares, niños.

\section{Introducción}

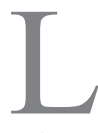

as infecciones osteo-articulares son una patología relativamente frecuente en pediatría, con una incidencia de 1: 1.000 a 1: 20.000 individuos para osteomielitis y 5,5 a 12: 100.000 para artritis séptica y comprenden tres entidades: artritis séptica, osteomielitis y la combinación de ambas ${ }^{1-2}$.

La epidemiología de estas infecciones está cambiando, principalmente a causa de que Staphylococcus aureus resistente a meticilina de la comunidad (SARM-Co) es el agente más frecuente en algunos países de Latinoamérica como Argentina y Uruguay, por ejemplo ${ }^{2-6}$. Aunque tradicionalmente considerado un patógeno nosocomial, SARM-Co se ha convertido ahora en una causa importante de infecciones en la comunidad, con pocos o ningún vínculo con centros de salud ${ }^{2-3}$. Su virulencia puede estar relacionada con su capacidad de producir toxinas, como la Panton-Valentine leucocidina (PVL), que tiene una capacidad única para destruir a los leucocitos, lo que resulta en la evasión bacteriana de la función bactericida de los mismos. Aunque inicialmente asociada con infecciones de la piel, recientemente se ha demostrado que la toxina PVL juega un papel esencial en la patogénesis de las infecciones invasoras, tales como neumonía necrosante, artritis y osteomielitis ${ }^{2-3-7}$. En algunos países de Latinoamérica, tal como Uruguay, se reportó un aumento de la incidencia y gravedad de las infecciones osteo-articulares agudas en los niños después de la emergencia de SARM$\mathrm{Co}^{3-4-8-9}$. Esta situación nos llevó a realizar este estudio para describir las características epidemiológicas, clínicas, microbiológicas y de evolución de los niños con infecciones osteo-articulares en un país con alta incidencia de SARM-Co en los últimos años ${ }^{9-10}$.

\section{Material y Métodos}

La incorporación de pacientes (p) para este estudio descriptivo, prospectivo, se llevó a cabo entre diciembre de 2011 y diciembre de 2013, en el Hospital de Pediatría Dr. Juan P. Garrahan, centro de referencia pediátrico terciario de Buenos Aires, Argentina.

Criterios de inclusión: Se incluyeron en el estudio to-
Hospital J P Garrahan, Buenos Aires, Argentina (MTR, GB, RB, $S G, C S, A B, G P, J P)$. Universidad Abierta Interamericana (UAI). CABA, Argentina (RL).

Los autores no presentan conflictos de intereses a declarar. No se recibió financiación para la elaboración del trabajo.

Recibido: 8 de enero de 2015 Aceptado: 7 de mayo de 2015

Correspondencia a: María Teresa Rosanova margris2@yahoo.com.ar 
dos los niños $\geq 3$ meses de edad que presentaban síntomas sugerentes de infección osteo-articular aguda, con menos de dos semanas de evolución.

\section{Criterios de exclusión}

Lactantes de menos de 3 meses; presencia de enfermedades subyacentes y/o intervenciones traumatológicas previas; cuadro clínico de infección osteo-articular de más de dos semanas de evolución; espondilodiscitis.

El estudio fue aprobado por el comité científico del hospital y se requirió el consentimiento informado de los padres.

El diagnóstico etiológico se basó en el cultivo de líquido articular, los hemocultivos y/o biopsias óseas mediante aspiración con aguja fina y/o drenaje quirúrgico, de acuerdo a cada caso individual.

Todas las muestras articulares u óseas se inocularon sistemáticamente en frascos de hemocultivo y se realizaron dos hemocultivos de cada $\mathrm{p}$ antes de iniciar el tratamiento con antimicrobianos.

Los cultivos fueron procesados de acuerdo con las directrices del Instituto de Normas Clínicas y de Laboratorio $(\mathrm{CLSI})^{11}$. Staphylococcus aureus se identificó mediante las pruebas microbiológicas habituales. La sensibilidad a meticilina se determinó mediante pruebas de difusión con discos de oxacilina de $1 \mu \mathrm{g}$ y de cefoxitina de $30 \mu \mathrm{g}$, en agar de Müeller Hinton (MHA) con incubación a 37 ${ }^{\circ} \mathrm{C}$ durante $24 \mathrm{~h}$. La detección de halos menores de 20 mm se consideró como resistencia. Se realizó detección selectiva en placa de MHA con 4\% de cloruro de sodio y $6 \mu \mathrm{g} / \mathrm{ml}$ de oxacilina, con inóculo de 100.000 ufc. Cualquier desarrollo se consideró resistente a meticilina. No se realizaron pruebas para detectar la producción de Panton-Valentine leucocidina.

Las infecciones osteo-articulares por SARM-Co se clasificaron de acuerdo a las definiciones del $\mathrm{CDC}^{12}$.

\section{Análisis estadístico}

Se adoptó a la mediana (Md) y al rango (r) como medidas de posición y dispersión. Las frecuencias se expresaron en porcentaje (\%). Las diferencias se consideraron significativas cuando $\mathrm{p}<0,05$ (prueba t de Student o Ude Mann-Whitney, según correspondiera).

\section{Protocolo de manejo}

Los $\mathrm{p}$ fueron tratados con esquemas antimicrobianos empíricos, inicialmente por vía intravenosa. De primera elección se utilizó clindamicina a una dosis de $30 \mathrm{mg} / \mathrm{kg} /$ día. El esquema empírico se adecuó luego, acorde a la identificación del agente etiológico y a su susceptibilidad in vitro.

El curso de la enfermedad se controló mediante la velocidad de sedimentación globular (ESD) y la proteína $\mathrm{C}$ reactiva $(\mathrm{PCR})$ cuantitativa en suero en el primer día de ingreso, a las 48 h, 7 días y cada semana hasta la normalización.

Para la ESD, se consideró elevado a un valor $>20$ $\mathrm{mm} / \mathrm{h}$. El valor de PCR fue determinado por nefelometría. Valores menores o iguales de $5 \mathrm{mg} / \mathrm{L}$ se consideraron normales.

En nuestra institución, para minimizar los riesgos asociados con la terapia antimicrobiana parenteral a largo plazo, se indica una secuencia de fármacos por vía intravenosa seguido de tratamiento antimicrobiano oral (conocido como terapia secuencial parenteral-oral). El pasaje a la vía oral se hizo en base a la ausencia de estado toxi-infeccioso y buena tolerancia oral, independientemente del valor de PCR o ESD. El antimicrobiano utilizado para la vía oral se adecuaba al microorganismo hallado y su sensibilidad. La dosis oral de cotrimoxazol era de $12 \mathrm{mg} / \mathrm{kg} /$ día de trimetoprim repartida cada $12 \mathrm{~h}$ y la dosis oral de clindamicina $30 \mathrm{mg} / \mathrm{kg} /$ día cada $8 \mathrm{~h}$.

Duración total del tratamiento: Dependiendo de la normalización de los valores de PCR, la duración total mínima del tratamiento fue de dos semanas para artritis y de tres para osteomielitis.

Como puntos finales de interés se consideró a la recuperación completa de los p sin recidivas ni secuelas durante al menos tres meses después del alta hospitalaria y a la ocurrencia de muerte durante la hospitalización.

\section{Resultados}

Se incluyeron $40 \mathrm{p}$ y todos completaron el estudio. De ellos, 20 p (50\%) tenían artritis, 17 p (43\%) osteomielitis y 3 p (7\%) osteo-artritis. Las características demográficas de los p se muestran en la Tabla 1. La edad fue de (Md) 90 meses (r: 3-186 m). El sitio más común de infección fue la cadera (12 p -30\%), seguido de la rodilla (9 p -22\%).

Tabla 1. Características de los pacientes (n: 40 pacientes)

\begin{tabular}{|lc|}
\hline Características & Total \\
\hline Edad en meses (Md, r) & $90(3-186)$ \\
Sexo masculino (\%) & $27 \mathrm{p} \mathrm{(68)}$ \\
Tiempo de evolución (Md) & 3 días \\
Fiebre & $38 \mathrm{p} \mathrm{(95 \% )}$ \\
Localización & $12 \mathrm{p} \mathrm{(30 \% )}$ \\
Cadera & $9 \mathrm{p} \mathrm{(22 \% )}$ \\
Tobillo & $7 \mathrm{p} \mathrm{(18 \% )}$ \\
Tibia & $5 \mathrm{p} \mathrm{(12 \% )}$ \\
Rodilla & $7 \mathrm{p} \mathrm{(18 \% )}$ \\
Otros & $30 \mathrm{p} \mathrm{(75 \% )}$ \\
Cultivos positivos & \\
\hline Md: mediana, r: rango, p: pacientes.
\end{tabular}


El valor de PCR (Md) al momento de la admisión fue de $50 \mathrm{mg} / \mathrm{L}$ (r: 26,3-86,5) que se normalizó dentro de los (Md) 7 días (r: 1-60 d). El nivel de ESD al ingreso fue de (Md) $75 \mathrm{~mm} / \mathrm{h}$ (r: 50-102) y se normalizó hacia los (Md) 28 días (r: 14-30 d). Los cultivos fueron positivos en 30 p (75\%): SARM-Co se aisló en 19 p; S. aureus sensible a meticilina (SASM) en $6 \mathrm{p}$ y otros microorganismos, en $5 \mathrm{p}$ (Streptococcus pyogenes: 3 p, Streptococcus pneumoniae: 1 p y Neisseria meningitidis $1 \mathrm{p}$ ). Los hemocultivos fueron positivos en $16 \mathrm{p} \mathrm{(40 \% ).} \mathrm{Todas} \mathrm{las} \mathrm{cepas} \mathrm{de} \mathrm{SARM-Co}$ fueron sensibles a cotrimoxazol y sólo 2 cepas fueron resistentes a clindamicina. La duración del tratamiento parenteral fue de (Md) 7 días (r: 5-10d) y la duración total del tratamiento fue de (Md) 28 días (r: 21-40 d); la estancia hospitalaria fue de (Md) 7 días (r: 3 a 10 d). Los p fueron seguidos durante (Md) 12 meses, durante los cuales 1 paciente recayó y 3 presentaron secuelas.

Comparando las principales características clínicas y de laboratorio de $\mathrm{p}$ con cultivos positivos para SARM-Co versus el resto de ellos, la PCR inicial fue mayor (Md 76 vs $50, \mathrm{p}<0,02$ ), la normalización se produjo después (Md 14 días vs 7 días, $\mathrm{p}<0,03)$ y la duración del tratamiento fue mayor (Md 32 días vs 23, p < 0,004). La estancia hospitalaria se prolongó pero sin alcanzar diferencias estadísticamente significativas (Md 9 días vs $7, \mathrm{p}=0,12$ ) (Figura 1).

Se realizó drenaje quirúrgico a todos los p con SARMCo. Las secuelas estuvieron presentes en 3 de ellos (acortamiento de la longitud del miembro afectado) y 1 , recayó. Ninguno falleció.

\section{Discusión}

En nuestro país, así como en varias partes del mundo, la epidemiología de las infecciones osteoarticulares ha cambiado debido a la aparición de SARM-Co ${ }^{9-10}$. Los cultivos suelen ser positivos en 40 a $60 \%$ de los casos de infecciones óseas y articulares. En $75 \%$ de nuestros $\mathrm{p}$ hubo aislamiento microbiológico y SARM-Co fue el agente etiológico más frecuente, coincidiendo con varios informes de la literatura médica ${ }^{9-13-14}$.

La PCR en suero desafía la posición tradicional de ESR para el diagnóstico y el seguimiento de las infecciones osteo-articulares de la infancia. Sus niveles séricos son más sensibles como prueba diagnóstica, útiles en el seguimiento y se determinan fácilmente ${ }^{15}$. La disminución de los niveles de PCR generalmente sugiere una respuesta favorable al tratamiento. Dado que la ESD aumenta rápidamente pero disminuye mucho más lentamente que el nivel de PCR, suele ser menos útil en el seguimiento de la evolución de la enfermedad.

Las infecciones óseas y articulares por SARM-Co provocan elevaciones mayores en el nivel de PCR y la

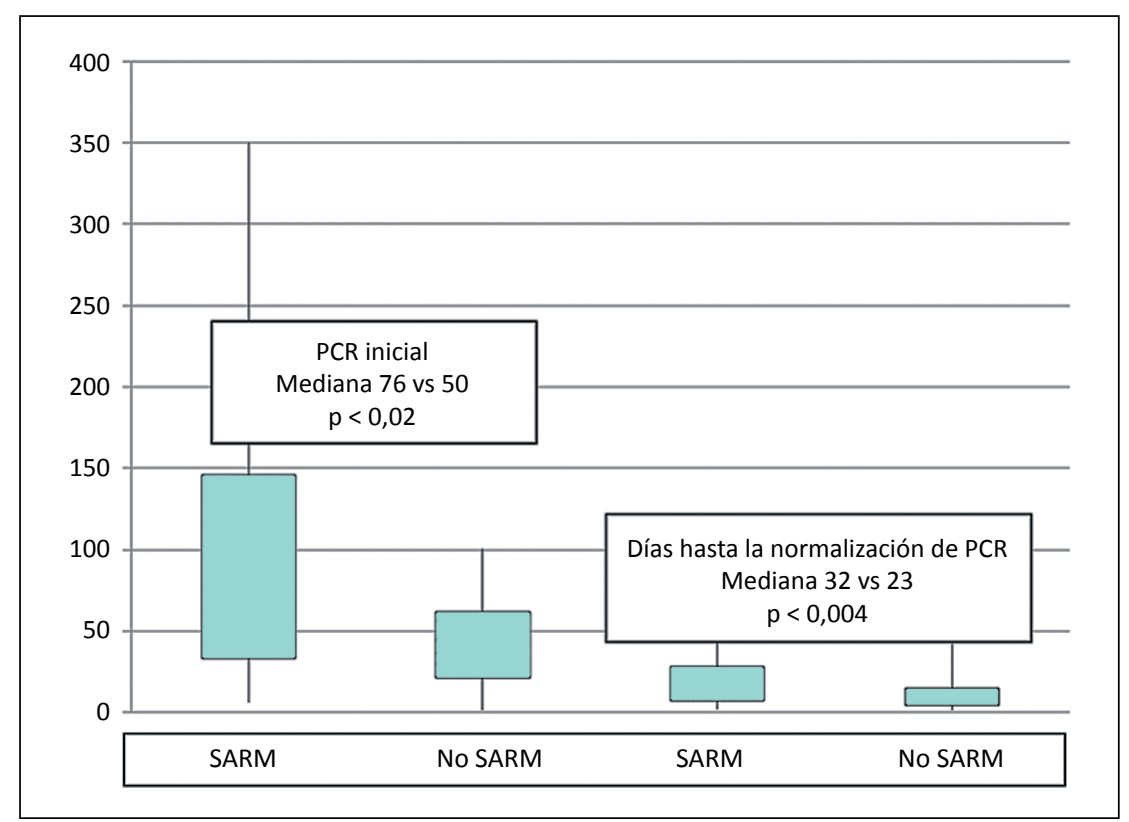

Figura 1. PCR inicial y días hasta la normalización SARM-Co y no-SARM.

normalización es más tardía, lo que coincide con lo visto en esta serie ${ }^{16}$.

El desbridamiento quirúrgico y el drenaje son la base del tratamiento de las infecciones osteo-articulares por SARM-Co y deben realizarse siempre que sea posible ${ }^{16}$.

La vía de administración óptima de los antimicrobianos no se ha establecido claramente. En estas infecciones, el tratamiento parenteral inicial seguido de tratamiento oral ha sido utilizado por diferentes autores con buenos resultados ${ }^{17,18}$. Cotrimoxazol, vancomicina y clindamicina son antimicrobianos útiles para tratar infecciones por SARM-Co. La tasa de resistencia a cotrimoxazol en cepas de SARM-Co es baja. Tampoco existen informes de cepas resistentes a vancomicina en nuestro medio 9 . Se ha publicado una tasa de resistencia variable a clindamicina. En un estudio multicéntrico en nuestro país, fue de 10\%; dato muy importante pues, según la recomendación de expertos, clindamicina es un antimicrobiano útil para el tratamiento empírico de estas infecciones, salvo que la tasa de resistencia a este antimicrobiano sea mayor a $15 \%$ en el área de trabajo ${ }^{9-10}$. Según los datos precedentes, clindamicina podría utilizarse como tratamiento empírico en estos niños como en esta serie donde fue el antimicrobiano empírico intravenoso de elección, incluso en $\mathrm{p}$ con bacteriemia.

La experiencia con cotrimoxazol para el tratamiento de infecciones por SARM-Co en niños es escasa y surge de informes aislados ${ }^{20}$. Trece de los diecinueve $\mathrm{p}$ con aislamiento de SARM-Co completaron el tratamiento oral con este 
fármaco sin evidencia de efectos adversos que obligaran a la suspensión de la misma y con buena evolución clínica. La duración total del tratamiento para las infecciones osteo-articulares en pediatría es un tema de controversia y debe ser individualizada. Las guías de práctica clínica actuales de la IDSA (Infectious Diseases Society of America), recomiendan un mínimo de 4 y 6 semanas de medicación para los niños con artritis aguda y osteomielitis, respectivamente. Con respecto al tiempo de tratamiento intravenoso su duración es variable según los centros, pero con tendencia a su reducción como en nuestro centro y en el trabajo de Prado y col $^{1}$, que redujeron a una semana el tratamiento intravenoso, seguido por tratamiento oral con buenos resultados. En p cuidadosamente seleccionados, el tiempo total del tratamiento podría acortarse si se normaliza la PCR. Esto ha sido implementado sobre todo en infecciones por SAMS ${ }^{13}$.

En esta serie, un paciente tuvo una recaída de la osteomielitis por SARM-Co después de un curso corto de 21 días de tratamiento y a pesar de haber presentado dos valores normales de PCR antes de la interrupción del tratamiento.

En comparación con SASM, SARM-Co es más agresivo en infecciones óseas y articulares y frecuentemente se asocia con trombosis venosa profunda, embolia pulmonar séptica o ambos ${ }^{3-14-17-20}$. En esta serie de p no encontramos este tipo de complicaciones graves. Sin embargo, la normalización de la PCR fue más tardía, lo que conllevó a una mayor duración del tratamiento antimicrobiano total y a la prolongación de la estancia hospitalaria. La mayor duración del tratamiento y el requerimiento de drenaje han sido reportados en la literatura de las infecciones osteo-articulares por SARM-Co, lo que corrobora este reporte $^{3-20}$.

En SARM-Co, las secuelas son más frecuentes. En nuestra serie, tres $\mathrm{p}$ con aislamiento de SARM-Co tuvieron secuelas que representaron acortamiento de los miembros afectados. Un p recayó con aislamiento de SARM-Co con la misma sensibilidad que el inicial.

Los resultados de este estudio intentan alertar al médico clínico en el manejo de niños con infecciones óseas y articulares donde, de acuerdo a la epidemiología local, debe considerarse siempre en el tratamiento empírico a SARM-Co. El rápido reconocimiento de los cambios en la epidemiología de las infecciones de huesos y articulaciones resultará en un tratamiento empírico más adecuado, ofreciendo una cobertura inicial para este patógeno para reducir la incidencia de complicaciones graves.
Las limitaciones del estudio incluyen el pequeño tamaño de muestra, que hace inviable crear un modelo de factores de riesgo para recaída y secuelas y una comparación exacta entre los distintos agentes etiológicos. $\mathrm{Su}$ diseño no es apropiado para evaluar la efectividad de los planes de tratamiento aplicados.

\section{Conclusiones}

SARM-Co fue la causa principal de las infecciones osteo-articulares y se asoció con mayor PCR al ingreso, normalización tardía y mayor duración del tratamiento. Es probable que el tratamiento empírico de las infecciones osteo-articulares en nuestro medio deba incorporar siempre un antimicrobiano con cobertura para SARM-Co.

Las complicaciones, requisitos de drenaje y secuelas fueron más comunes en esos p. Por el momento, puede postularse que en las infecciones donde se aísle SARM-Co, no deberían implementarse cursos cortos de tratamiento.

\section{Resumen}

Introducción: Las infecciones osteo-articulares (IOA) son relativamente comunes en los niños, siendo la infección por Staphylococcus aureus resistente a meticilina de la comunidad (SARM-Co) una de las más frecuentes. Objetivo: Evaluar los datos epidemiológicos, características clínicas, microbiológicas y de evolución en niños con IOA. Métodos: Estudio descriptivo prospectivo. Resultados: Se incluyeron 40 pacientes (p). Los cultivos fueron positivos en $30 \mathrm{p}$ (75\%). Se aisló SARM-Co en $19 \mathrm{p} ;$ S. aureus sensible a meticilina en 6 p; otros microorganismos en 5 p. La duración del tratamiento fue de 28 días Md (r: 21-40 d). En los p con cultivos positivos para SARM-Co, la PCR inicial fue mayor (Md 76 vs $50 \mathrm{mg} / \mathrm{L}$, $\mathrm{p}<0,02$ ), la normalización se produjo después (Md 14 días vs 7 días, $\mathrm{p}<0,03$ ) y la duración del tratamiento ( $\mathrm{Md}$ 32 días vs $23, p<0,004)$, así como la estancia hospitalaria (Md 9 días vs 7, p =0,12) fueron más prolongados. En la evolución 1 p recayó y 3 tuvieron secuelas; en todos se aisló SARM-Co. Conclusión: SARM-Co fue la causa más frecuente de las IOA y se asoció con mayor valor de PCR al ingreso, normalización tardía, mayor duración del tratamiento, y complicaciones. 


\section{Referencias bibliográficas}

1.- Prado A, Lizama M, Peña A, Valenzuela C, Viviani T. Tratamiento intravenoso inicial abreviado en 70 pacientes pediátricos con infecciones osteo-articulares. Rev Chilena Infect 2008; 25: 30-6.

2.- Zaoutis T E, Toltzis P, Chu J, Abrams T, Dul M, Kim J, et al. Clinical and molecular epidemiology of community-acquired methicillin-resistant Staphylococcus aureus infections among children with risk factors for health care-associated infection: 2001-2003. Pediatr Infect Dis J 2006; 25: 343-8.

3.- Arnol S, Elias D, Buckingham S, Thomas E D, Novais E, Arkader A, et al. Changing patterns of acute hematogenous osteomielitis and septic arthritis. J Pediatr Orthop 2006; 26: 703-8.

4.- Kaplan S. Community-acquired methicillinresistant Staphylococcus aureus infections in children. Semin Pediatr Infect Dis 2006; 17: 113-9.

5.- Francis J S, Doberty M C, Lopatin U, Johnston C P, Sinha G, Ross T, et al. Severe communityonset pneumonia in healthy adults caused by methicillin resistant $S$. aureus carrying the Panton Valentine leukocidin genes Clin Infect Dis 2005; 40: 100-7.

6.- Galeana Villar A. Infección por Staphylococcus aureus meticilino-resistente adquirido en la comunidad. Arch Pediatr Uruguay 2003; 74 : 26-9.

7.- Vardakas K Z, Kontopidis I, Gkegkes I D, Rafailidis P I, Falagas M E. Incidence, characteristics, and outcomes of patients with bone and joint infections due to community- associated methicillin-resistant Staphylococcus aureus: a systematic review. Eur J Clin Microbiol Infect Dis 2013; 32: 711-21.

8.- Stoesser N, Pocock J, Moore C, Soeng S, Hor P, Sar P, et al. The epidemiology of pediatric bone and joint infections in Cambodia, 2007-11 J, Trop Pediatr 2013; 59: 36-42.

9.- Morrison M A, Hageman J C, Klevens R M. Case definition for community-associated methicillin-resistant Staphylococcus aureus. J Hosp Infect 2006; 62: 241-6.

10.- Paganini H, Della Latta P, Muller Opet B, Ezcurra G, Uranga M, Aguirre C, et al. Community-acquired methicillin-resistant Staphylococcus aureus infections in children: multicenter trial. Arch. Argent. Pediatr 2008; 106: 397-403.

11.- Egea A L, Gagetti P, Lamberghini R, Faccone D, Lucero C, Vindel A, et al. New patterns of methicillin-resistant Staphylococcus aureus (MRSA) clones, community-associated MRSA genotypes behave like healthcare-associated MRSA genotypes within hospitals, Argentina. Int J Med Microbiol 2014; 304: 1086-99.

12.- CLSI, 2013. Performance standards for antimicrobial susceptibility testing. CLSI approved standard M100-S23. Clinical and Laboratory Standards Institute, Wayne, PA.

13.- Liu C, Bayer A, Cosgrove S, Daum R S, Fridkin S K, Gorwitz R J, et al. Clinical Practice Guidelines by the infectious Diseases Society of America for the treatment of methicillinresistant $S$. aureus infections in adults and children: Executive Summary. Clin Infect Dis 2011; 52: 285-92.

14.- Pääkkönen M, Kallio M J, Kallio P E,
Peltola H. Shortened hospital stay for childhood bone and joint infections: analysis of 265 prospectively collected culture-positive cases in 1983-2005. Scand J Infect Dis 2012; 44: 683-8.

15.- Kini A, Shetty V, Kumar A, Shetty S M, Shetty A. Community-associated, methicillinsusceptible, and methicillin-resistant Staphylococcus aureus bone and joint infections in children: experience from India. J Pediatr Orthopedic 2013; 22: 156-66.

16.- Arnold J, Cannavino M, Westley B, Westley B, Miller T C, Riffenburgh R H, et al. Acute bacterial osteoarticular infections: Eighth year analysis of C-Reactive Protein for oral step down therapy. Pediatrics 2012; 130: 821-9.

17.- Crary S E, Buchanan G R, Drake C E, Journeycake J M. Venous thrombosis and thromboembolism in children with osteomyelitis. J Pediatr 2006; 149: 537-41.

18.- Pääkkönen $M$, Peltola $H$. Bone and joint infections. Pediatr Clin North Am 2013; 60: 425-36.

19.- Kaplan S L. Recent lessons for the management of bone and joint infections. J Infect 2014; 68: S51-6.

20.- Martínez-Aguilar G, Hammerman W A, Mason E O Jr, Kaplan L. Clindamycin treatment of invasive infections caused by communityacquired, methicillin-resistant and methicillinsusceptible Staphylococcus aureus in children. Pediatr Infect Dis J 2003; 22: 593-6.

21.- Messina A F, Namtu K, Guild M, Dumois J A, Berman D M. Trimethoprim-sulfamethoxazole therapy for children with acute osteomyelitis. Pediatr Infect Dis J 2011; 30: 1019-21. 